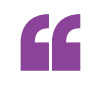

the reduction in

AD pathology was dependent on PINK 1 kinase activity

ALZHEIMER DISEASE

\title{
PINK1 rescues pathology in Alzheimer disease
}

Decreased expression of PTENinduced putative kinase 1 (PINK1), an important effector of mitochondrial integrity and function, is associated with Alzheimer disease (AD) pathology, according to a new study reported in Brain. The results present a new pathway that could potentially be targeted to treat $\mathrm{AD}$.

"Mitochondrial dysfunction and synaptic failure are prominent and early pathological features of AD," explains corresponding author Shirley ShiDu Yan. "PINK1 is critical for the maintenance of mitochondrial integrity and function by promoting the removal of damaged mitochondria via mitophagy; however, the role of PINK1 in the pathogenesis of $\mathrm{AD}$ is largely unknown."

Interestingly, PINK1 is downregulated in the brains of patients with $\mathrm{AD}$ and in the mAPP transgenic mouse model, which expresses human amyloid- $\beta(A \beta)$. To investigate the role of PINK1, the researchers used viral vectors to express either wild-type PINK1 or a kinase-inactive mutant form of PINK1 in the hippocampus of mAPP mice.

"Most intriguingly, restoring and enhancing PINK1 expression and activity not only improved mitochondrial and cognitive function, but also robustly attenuated cerebral and mitochondrial $A \beta$ production and accumulation," comments Yan. Notably, the reduction in AD pathology was dependent on PINK1 kinase activity, as overexpression of kinase-inactive PINK1 did not reduce $A \beta$ accumulation, mitochondrial defects or synaptic abnormalities in $\mathrm{mAPP}$ mice. In addition, PINK1-deficient mAPP mice showed accelerated $A \beta$ accumulation and early deficits in cognitive and mitochondrial functions, which further highlights the importance of PINK1 signalling in AD.

“The validation of PINK1's role in $\mathrm{AD}$ is expected to assist in drug development to limit $A \beta$ pathology and lessen mitochondrial and synaptic injury, which represents a novel therapeutic approach to halt $\mathrm{AD}$

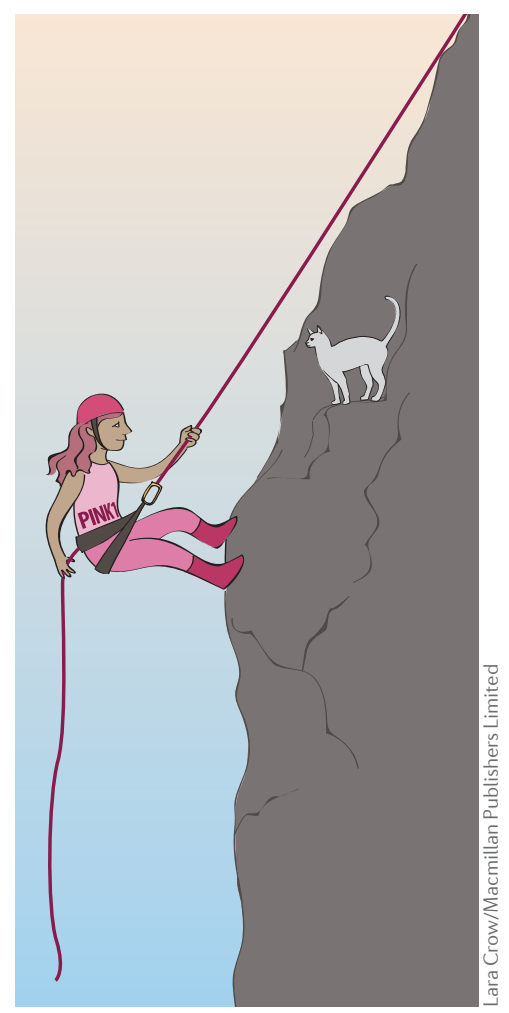

at a very early stage by eliminating $\mathrm{A} \beta$ accumulation and powering up mitochondrial function," concludes Yan.

Shimona Starling

ORIGINAL ARTICLE Du, F. et al. PINK1 signalling rescues amyloid pathology and mitochondrial dysfunction in Alzheimer's disease. Brain http:// dx.doi.org/10.1093/brain/awx258 (2017) 